\title{
A new cross sectional echocardiographic method for estimating the volume of large pericardial effusions
}

\author{
Ivan A D’Cruz, Philip K Hoffman
}

\begin{abstract}
Objective-To evaluate a new cross sectional echocardiographic method for estimating the volume of pericardial effusions.
\end{abstract}

Design-The volume of pericardial fluid removed by surgical drainage or paracentesis was compared with the volume estimated by the echocardiographic method. The pericardial sac volume and cardiac volume were calculated by applying the formula for the volume of a prolate ellipse $(\pi \times 4 / 3 \times L / 2$ $\times D 1 / 2 \times D 2 / 2)$ where $L$ is the major axis and $D 1$ and $D 2$ are the minor axes. The pericardial fluid volume was calculated as the pericardial sac volume minus the cardiac volume.

Patients-13 patients with 14 large pericardial effusions (one recurrence) all of whom had tamponade and cross sectional echocardiography just before therapeutic full drainage of the effusion.

Results-The volumes of pericardial fluid drained ranged from 0.5 to $2 \cdot 11$. The correlation between these actual volumes and the volumes estimated by echocardiography was excellent $(\mathbf{r}=0.97)$; the correlation was good in four patients with intrapericardial adhesions.

Conclusions-Because of certain approximations in measuring quantity of pericardial fluid drained, the echocardiographic estimations cannot be claimed to be definite. The data, however, indicate that the echocardiographic method is sufficiently reliable to provide useful estimates for practical clinical purposes.

\footnotetext{
Medical College of Georgia and VA Medical Center, Augusta, Georgia, USA

I A D'Cruz

Medical College of Georgia, USA P K Hoffman

Correspondence to Dr Ivan A D'Cruz Cardiology Section, VA Cardiology Section, VA Medical Center, Augusta, Georgia 30910, USA

Accepted for publication 27 June 1991
} effusions as small, moderate, or large ${ }^{3}$ on the basis of the width of the sonolucent pericardial space posterior and anterior to the heart on the $M$ mode echocardiogram. Attempts to
The pioneering work of Feigenbaum et al cedure of choice for imaging exaggerated cardiac motion in large pericardial effusions. ${ }^{2}$

Since the landmark paper by Horow $a l$, it has been common echocardiographic estimate the quantity of pericardial fluid from the $M$ mode echocardiogram by cubing the widths of the pericardial sac and of the heart and then subtracting the heart volume from the pericardial sac have yielded conflicting results. Correlation between such estimates of effusion and the actual quantity of pericardial fluid removed at cardiothoracic surgery was reported as good by Horowitz et $a l^{3}$ and by Parameswaran and Goldberg ${ }^{4}$ but poor by Prakash et al. ${ }^{5}$

We evaluated a simple cross sectional echocardiographic method to estimate the volume of large pericardial effusions.

\section{Patients and methods}

Our series of 13 patients all had large pericardial effusions and clinical evidence of tamponade. Six other patients were excluded because the echocardiograms were of inadequate quality. Haemodynamic evidence of tamponade was available in eight. All pericardial effusions were drained therapeutically, either surgically (subcostal pericardial window) in seven instances or percutaneously with tube drainage in seven instances. In 10 patients the pericardial effusion was drained within three hours of the echocardiogram; in the other four the interval was 4-12 hours. All the effusions were drained as completely as possible-that is, until no more fluid was obtainable. In one patient, a large pericardial effusion recurred five months after complete initial drainage, and was drained again.

The aetiology of pericardial effusions was as follows: coronary bypass surgery (three), aortic valve replacement on anticoagulant (two), pulmonary embolism on anticoagulant (one), lung carcinoma (two), renal cell carcinoma (one), collagen disease (one), $E$ coli endocarditis (one), aetiology uncertain (two). Echocardiography was performed with Hewlett-Packard (77020 A) equipment and a $2.5 \mathrm{MHz}$ phased array transducer. All measurements were made directly on the videoscreen image by the computerised software measurement package; and then each video image together with the dimensions measured and digital readout was documented on hard copy. Figure 1 shows the cross sectional echocardiographic dimensions that we measured. 
Apical four chamber view

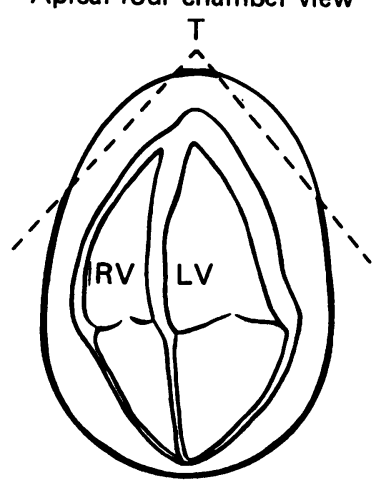

Parasternal short axis view

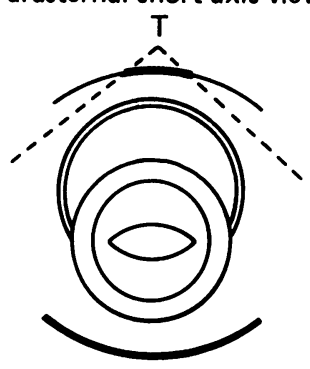

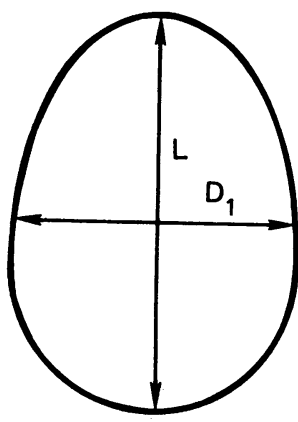

Pericardium

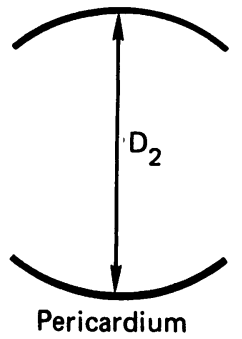

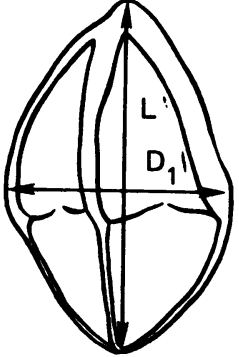

Heart

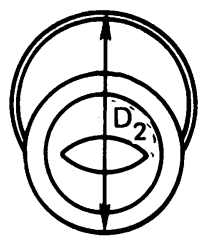

Heart

Figure 1 Diagram showing how the major axis $(L)$ and minor axes D1 and D2 were measured in the pericardium and heart in the apical four chamber view and parasternal short axis view. $T$, transducer; $R V$, right ventricle; $L V$, left ventricle.

\section{PERICARDIUM}

We measured the maximal long axis dimension (L) and maximal transverse dimension (D1) in the apical four chamber view and maximal anteroposterior dimension (D2) in the parasternal long or short axis view at the mitral leaflet level.

\section{HEART}

Length ( $\mathrm{L})$ (maximal long axis dimension) was measured from the tip of ventricular apex to the cephalad border of atria at the junction of right and left atrium in the apical four chamber view; D1 (maximal transverse dimension) was measured in the apical four chamber view, which was usually obtained at the level of the mitral and tricuspid leaflets, 1 to $3 \mathrm{~cm}$ on the ventricular side of the atrioventricular valve annuli; D2 (maximal anteroposterior dimension in the parasternal long or short axis view) was measured at the mitral

Figure 2 Correlation between amount of pericardial fluid drained and echocardiographically estimated pericardial effusion.

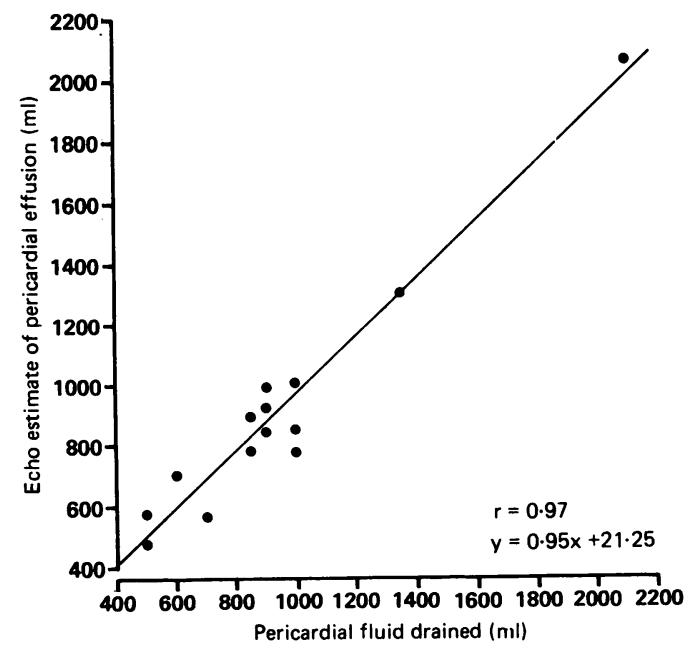

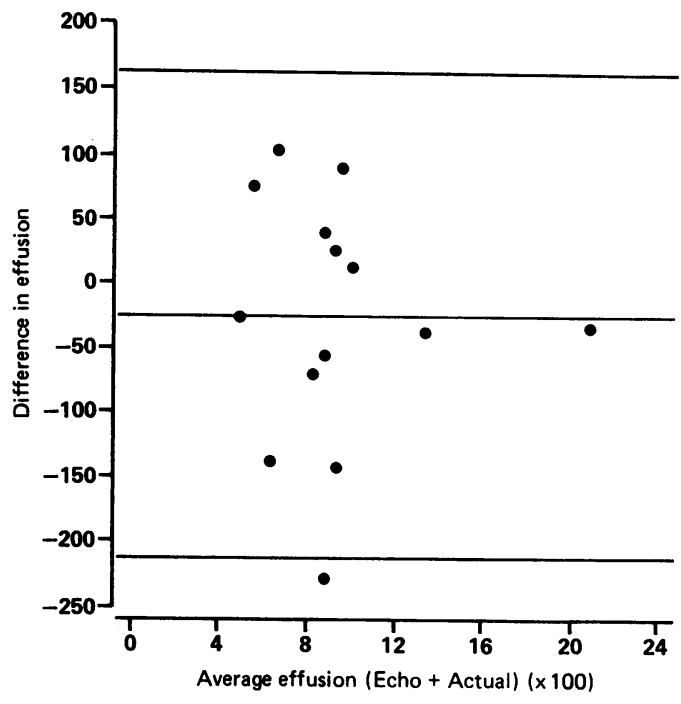

Figure 3 Agreement between the echocardiographically estimated and drained volumes of pericardial effusions calculated by the Bland and Altman method. ${ }^{6}$

leaflet level. Three to six measurements were made of each dimension on different cardiac cycles and the mean of each dimension was calculated. All measurements were made at the onset of the QRS complex. Pericardial measurements were made from inner border to inner border. Cardiac measurements were made from epicardium to epicardium. Care was taken to avoid side-lobe artefacts simulating epicardial echoes. The observer making the echocardiographic measurements was unaware of the quantity of pericardial fluid drained. The volumes of the pericardial sac and heart were calculated by applying the formula for volume of a prolate ellipse: $\pi \times 4 / 3 \times \mathrm{L} / 2 \times \mathrm{D} 1 / 2 \times \mathrm{D} 2 / 2$, where $\mathrm{L}$ is the major axis and D1 and D2 the two minor axes. The pericardial fluid volume was calculated as the pericardial sac volume minus cardiac volume.

\section{Results}

The echocardiographically estimated volume of pericardial fluid correlated well (fig 2) with the volume of pericardial fluid drained $(r=$ 0.97). Data from the two patients with the largest pericardial effusions made a large contribution. When the patient with the largest effusion $(2 \cdot 11)$ was excluded, the $r$ value fell to 0.93 , and when this patient and the patient with the next largest effusion ( 1.351$)$ were excluded, the $\mathrm{r}$ value fell to $\mathbf{0 \cdot 8 8}$.

The agreement between the two measurement methods was calculated by the Bland and Altman method. ${ }^{6}$ The limits of agreement for the volume of pericardial effusion (echocardiographic minus drained volume) were -211.72 and $161.86 \mathrm{ml}$ (fig 3).

The two patients in whom the echocardiographic method underestimated the amount of the pericardial fluid to the largest extent $(230$ and $144 \mathrm{ml}$ ) had the most conspicuous atrial compression, such that the atrial free walls appeared concave rather than convex on the cross sectional echocardiogram in the apical four chamber view.

Intrapericardial adhesions at the cardiac 
Figure 4 Cross sectional echocardiogram of a patient with a large pericardial effusion and tamponade. In the apical four chamber view (left) the dimensions $P-P$ are the major axis (L) and minor axis D2 of the pericardial sac; the corresponding cardiac dimensions are labelled $C-C$. Side lobe artefacts adjacent to the left ventricular and right ventricular free walls must be recognised. The parasternal short axis view (right) shows the minor axis D2 of the pericardal sac $(P-P)$ and the heart (C-C).

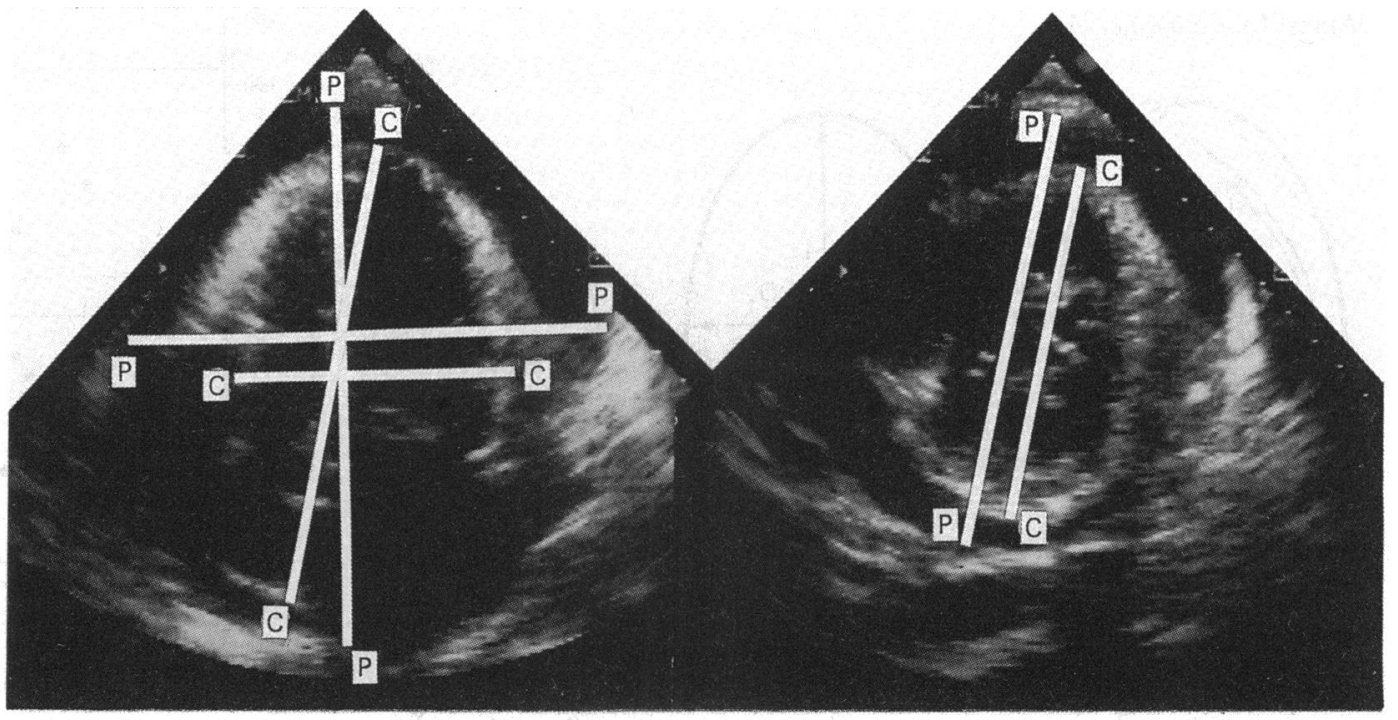

apex, with or without involvement of a small amount of the adjacent anterior wall, were seen on cross sectional echocardiography in three patients. In one other patient extensive pericardial adhesions had sealed off the whole anterior cardiac surface so that the large pericardial effusion was entirely loculated posteriorly; the echocardiographic estimate of the pericardial effusion $(1012 \mathrm{ml})$ was very close to the actual quantity of fluid drained (ll).

\section{Discussion}

Clinicians need to know the approximate volume of a pericardial effusion to help make management decisions about draining the effusion and discharging the patient from hospital and to ascertain whether the effusion is increasing, decreasing, or unchanged in quantity on serial echocardiograms.

At present pericardial effusions are commonly graded by size as small, moderate, or large. However, these terms have widely different meanings to different echocardiographers. For example, some regard pericardial effusions of $<500 \mathrm{ml}$ as small, those between 500 and $1000 \mathrm{ml}$ as moderate, and those $>1000 \mathrm{ml}$ as large ${ }^{7}$; Arvin described effusions of $150-400 \mathrm{ml}$ as moderate and those of 500 $2000 \mathrm{ml}$ as large ${ }^{8}$; Menapace regards effusions of $25-100 \mathrm{ml}$ as small, those of $100-500 \mathrm{ml}$ as moderate, and those $>500 \mathrm{ml}$ as large. ${ }^{9}$ Further ambiguity has been introduced by the use of terms such as "moderately large" or "moderate-to-large" effusions.

Unlike the left ventricle, the fluid-filled pericardial space between the heart and parietal pericardium does not conform to a simple geometric shape so that extrapolation of one or more dimensions of the pericardial space cannot be expected reliably to estimate the quantity of pericardial fluid. So another approach was attempted to estimate the total pericardial volume and the volume of the heart (from the $M$ mode echocardiogram by simple cubing of the short axis dimension) and subtracting the estimated cardiac volume from the total pericardial volume to obtain the volume of the met with qualified success ${ }^{34}$ but not for others. ${ }^{5}$ Horowitz et al found a correlation coefficient of 0.98 in 12 patients, but this fell to 0.41 when the two patients with largest effusions were excluded. ${ }^{3}$ Parameswaran and Goldberg reported a correlation coefficient of 0.78 in 23 patients. ${ }^{4}$

Cross sectional echocardiography of pericardial effusions of varying sizes showed that pericardial fluid was not uniformly distributed around the heart in circumcardiac effusions $^{11^{11}}$; it also showed an even greater asymmetry of pericardial fluid accumulation when there were intrapericardial adhesions or fluid loculations. ${ }^{12}{ }^{13} \mathrm{M}$ mode echocardiograms, which give an ice-pick view in the anteroposterior dimension only, might therefore be expected often to overestimate or underestimate pericardial effusion volume, especially since cubing of the dimensions (cardiac and pericardial) would magnify errors secondary to disparities in pericardial width. To our knowledge, few if any echocardiography laboratories use this $\mathbf{M}$ mode method to report volume estimates of pericardial effusions in routine clinical practice.

The pericardium is sometimes described as a conical flask-shaped structure with its base on the diaphragm and the great vessels emerging from the "mouth" of the flask. This may be true of the unopened fibrous pericardium as viewed in situ. When it is imaged by cross sectional echocardiography, however, the serous pericardial sac is ellipsoidal, whether or not it contains an effusion.

We assumed that the heart too is ellipsoidal and hence we used the same formula to estimate its volume.

In patients with conspicuous biatrial compression caused by tamponade, ${ }^{14}{ }^{15}$ which is particularly manifest at the onset of the QRS complex, the contour of the atria may be concave rather than convex so that the estimated cardiac volume is larger (and the quantity of pericardial fluid smaller) than the actual amount present. This is the most likely reason why our echocardiographic method underestimated the quantity of pericardial effusion in two patients. 
The volume of pericardial fluid estimated by our echocardiographic method correlated well with the actual quantity of fluid drained. Although the very high correlation coefficient $(0.97)$ was in part due to the two patients with pericardial effusions that were much larger than the rest, the correlation remained quite $\operatorname{good}(r=0.88)$ even when these two patients were excluded.

The presence of intrapericardial adhesions or loculation in four patients did not preclude a good correlation between the amount of pericardial fluid drained and our echocardiographic estimate. We have previously called attention to potential pitfalls in the $M$ mode grading of pericardial effusions by size in patients with intrapericardial adhesions. ${ }^{16}$

In a review article on echocardiography in pericardial disease, Schiller briefly mentioned having used cross sectional echocardiography in "a few" patients to measure the volume of pericardial contents. ${ }^{17} \mathrm{He}$ used a light-pen computational system to outline the borders of the pericardium and of the heart in the apical four chamber view. Volumes were computed electronically by an area-length single-plane algorithm. Schiller pointed out that an unavoidable shortcoming of this approach is that the pericardial border near the apical region cannot be visualised because it falls outside the narrow part of the fan-shaped sector image. However, non-visualisation of this portion of the pericardial outline does not interfere with measurement of the pericardial dimensions required for estimation of pericardial volume by our method (fig 4).

We are aware of certain shortcomings of this study, but believe them to be minor. The quantity of pericardial fluid was not measured precisely to the millilitre but "rounded off" to the nearest $50 \mathrm{ml}$. When the pericardial fluid was drained surgically we know that the entire pericardial contents were removed. We believe that the inevitable loss of a small amount of pericardial fluid (not included in the measured quantity) at the beginning of the drainage procedure was less than $50 \mathrm{ml}$ and often less than $25 \mathrm{ml}$. When the effusions were drained by paracentesis and tube drainage we may not have obtained and measured all the epicardial fluid. In nine of the patients we had echocardiograms in multiple views obtained immediately after drainage in six and within 18 hours in three. In all of these no pericardial effusion was visualised or only a small posterior pericardial pool no more than $5 \mathrm{~mm}$ deep and no more than $2.5 \mathrm{~cm}$ in diameter. We therefore think it reasonable to assume, for practical purposes, that all (or almost all) of the pericardial effusion had been drained off and measured. Our echocardiographic method does not take into account accumulation of fluid in the various minor pericardial recesses.

We thank Lelia Childers and Mary Walden for technical assistance, $\mathbf{R}$ Chris Hand and Mark Litaker for statistical assistance, Carol Brown for wordprocessing skills, and Russ Baltesar and Karen McBride for help in preparing the illustrations.

1 Feigenbaum $H$, Waldhausen JA, Hyde LP. Ultrasound diagnosis of pericardial effusion. JAMA 1965;191:107-10.

2 Feigenbaum H, Zaky A, Grabhorn L. Cardiac motion in patients with pericardial effusion: a study using ultrasound patients with pericardial effusion: a study

3 Horowitz MS, Schulz CS, Stinson EB, Harrison DC, Popp RL. Sensitivity and specificity of echocardiographic diag nosis of pericardial effusion. Circulation 1974;50:239-47.

4 Parameswaran R, Goldberg H. Echocardiographic quantiParameswaran R, Goldberg H. Echocardiographic quan
tation of pericardial effusion. Chest 1983;83:767-70.

5 Prakash R, Moorthy K, Delvicario L, Aronow WS. Reliability of echocardiography in quantitating pericar dial effusion: a prospective study. JCU 1977;5:398-402.

6 Bland JM, Altman DG. Statistical methods for assessing agreement between two methods of clinical measurement. Lancet 1986;i:307-10.

7 St John Sutton M, Oldershaw PJ, eds. Echocardiography and Doppler. New York: Blackwell, 1989:384

8 Arvan S. Echocardiography. New York: Churchill Livingstone, 1984:224.

9 Menapace FJ. Two-dimensional echocardiography in pericardial disease. In: Giuliani E, ed. Two-dimensional real-time ultrasonic imaging of the heart. Boston: Martinus Nijhoff, 1985:182.

10 Feigenbaum H. Echocardiography. 4th ed. Philadelphia: Lea and Febiger, 1986:554.

11 Martin RP, Rakowski $H$, French J. Localisation of pericardial effusion with wide-angle phased-array echocarcardial effusion with wide-angle phased-ar

12 Friedman MJ, Sahn DJ, Haber K. Two-dimensional echocardiography and B-mode ultrasonography for the diagnosis of loc

13 D'Cruz IA, Kensey K, Campbell C, Replogle R. Twodimensional echocardiography in cardiac tamponade following open-heart surgery. J Am Coll Cardiol 1985; 5:1250-2.

14 Kronzon I, Cohen ML, Winer HE. Diastolic atrial compression: a sensitive echocardiographic sign of cardiac tamponade. J Am Coll Cardiol 1983;2:770-5.

15 D'Cruz I, Callaghan W, Arensman F, Gross C. Left atria compression: a neglected sign of tamponade [abstract] $J$ Am Coll Cardiol 1988;11:84A

16 D'Cruz I, Prabhu R, Cohen HC, Glick G. Potential pitfalls in quantification of pericardial effusions by echocardioraphy. Br Heart J 1977;39:529-35.

17 Schiller NB. Echocardiography in pericardial disease. Med Clin N Am 1980;64:252-82. 